\title{
Flat OFC Generation Based on DPMZM Cascaded Dual-Parallel PolM with Frequency Multiplication Circuit
}

\author{
Xin WANG, Sijia LIU, Ximing WANG, Yingxi MIAO, Caili GONG, Yongfeng WEI ${ }^{1}$ \\ Department of Electronic Information Engineering, Inner Mongolia University, \\ Hohhot,010021, China
}

\begin{abstract}
An innovative scheme to generate the high-quality OFC based on DPMZM cascaded dual-parallel PolM with frequency multiplication circuit is proposed and demonstrated. In the scheme, 5 comb lines are generated in the firststage generator, and the comb line is expanded to 11 times in the second-stage generator. The theoretical model of the overall scheme is established and analyzed. In this scheme, 66-line OFC is generated and the flatness is $0.73 \mathrm{~dB}$, the side mode suppression ratio (SMSR) is $14.19 \mathrm{~dB}$, and the optical signal noise ratio (OSNR) is about $29 \mathrm{~dB}$.
\end{abstract}

Keywords. OFC generation, DPMZM, PolM, frequency multiplication circuit

\section{Introduction}

Microwave photonics has many unique superiorities, for instance the large bandwidth, low transmission loss, multiplexable and resistance to electromagnetic interference (EMI), and will be applied widely in radar technology, which can improve the performance of radar.

In microwave photonic radar system, high-quality multi-wavelength light sources are required, and the high-quality optical frequency comb (OFC) with high flatness, a large number of comb lines and the tunable frequency interval are considered as a research hotspot, and can be applied in WDM system [1], photonic filtering [2] and optical millimeter wave generating [3], etc. In the past decade, various schemes have been proposed for generating OFC, for instance the optical nonlinear effect method [4], the mode-locked laser method [5], the photoelectric oscillator method [6], and the external modulator method [7-10]. The method of generating OFC based on the external modulator has superiorities of stability, flexibility and adjustment, which has attracted great attention. In 2013, a novel approach to generating an OFC by applying a polarization modulator (PolM) and a polarizer was proposed which can generate 25-line OFC, and the flatness of OFC is $1 \mathrm{~dB}$ [8]. In 2014, a low-cost and simple OFC generation scheme was proposed by applying a dual-parallel Mach-Zehnder modulator (DPMZM) and generate 5-line and 7-line OFC with the flatness of $0.1 \mathrm{~dB}$ and $1 \mathrm{~dB}$, respectively [9].

\footnotetext{
${ }^{1}$ Corresponding author: Yongfeng Wei, Department of Electronic Information Engineering, Inner Mongolia University, Hohhot, 010021, China ; E-mail: wyfily@bupt.edu.cn .
} 
In 2015, a new scheme to generate OFC using one MZM and DPMZM which generate 9-line OFC, and the flatness is $0.8 \mathrm{~dB}$ [10].

In this scheme, a new scheme to generate the high-quality OFC is proposed and demonstrated. The result shows that 66 -line OFC is generated, the flatness is $0.73 \mathrm{~dB}$ and SMSR is $14.19 \mathrm{~dB}$.

\section{Scheme principle and analysis}

The schematic diagram of flat OFC generation based on DPMZM cascaded dual-parallel PolM with frequency multiplication circuit is shown in Figure 1.

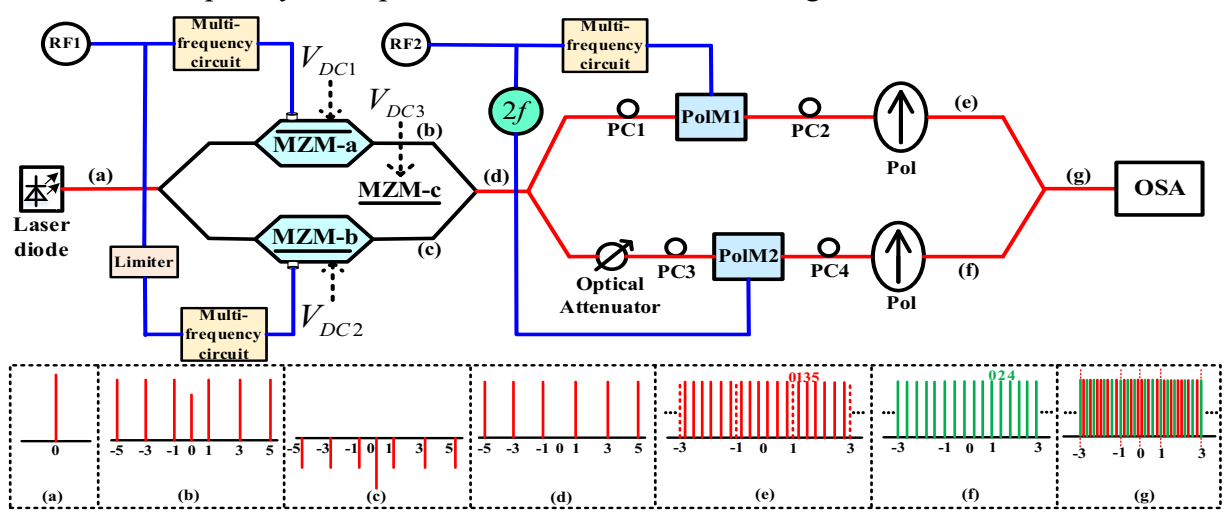

Figure 1. Schematic diagram of the flat OFC generation based on DPMZM cascaded dual-parallel PolM with frequency multiplication circuit.

Assuming the light wave launched from the laser diode can be expressed as $E_{i n}(t)=E_{c} e^{j \omega_{c} t}$. On the upper branch, the RF1 is injected into the frequency multiplication circuit. The schematic diagram of frequency multiplication circuit is shown in Figure 2. On the lower branch, the RF1 signal is limited by a limiter, and then injected into the frequency multiplication circuit.

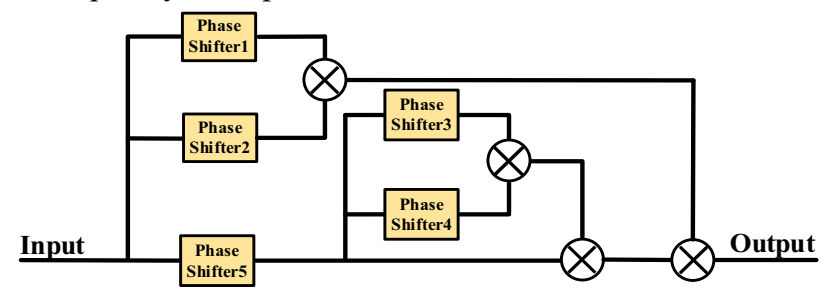

Figure 2. Schematic diagram of frequency multiplication circuit.

The phase shifts of electric frequency shifters are $\theta_{P S 1}=\pi / 3, \theta_{P S 2}=-\pi / 3$, $\theta_{P S 3}=\pi / 3, \theta_{P S 4}=-\pi / 3$ and $\theta_{P S 5}=\pi / 2$ respectively. The RF1 signal is $V_{\mathrm{RF} 1}(t)=V_{R F 1} \cos \left(\omega_{1} t\right)$, after frequency multiplication circuit, the output is:

$$
\begin{aligned}
V_{1}(t) & =V_{R F 1}^{5} \sin \left(\omega_{1} t+\frac{\pi}{3}\right) \cdot \sin \left(\omega_{1} t-\frac{\pi}{3}\right) \cdot \sin \left(\omega_{1} t-\frac{\pi}{3}\right) \cdot \cos \left(\omega_{1} t+\frac{\pi}{3}\right) \cdot \cos \left(\omega_{1} t\right) \\
& =\frac{V_{R F 1}^{5}}{16}\left[\cos \left(\omega_{1} t\right)+\cos \left(3 \omega_{1} t\right)+\cos \left(5 \omega_{1} t\right)\right]
\end{aligned}
$$


The output spectrogram is shown in Figure 3, assuming that $\omega=4 \mathrm{GHz}$.

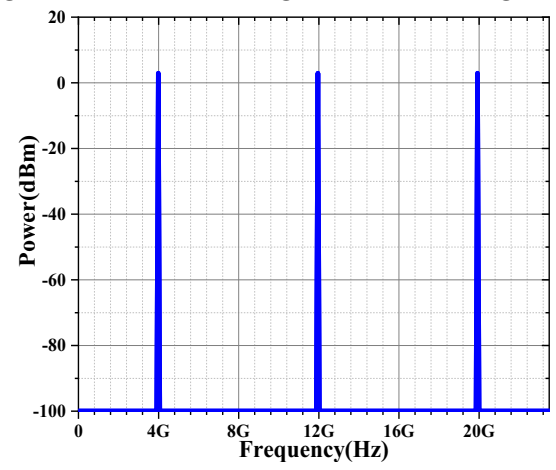

Figure 3. Electrical spectrum of the output signal.

The drive signals output by the frequency multiplication circuit are injected into DPMZM for modulation. MZM-a and MZM-b are biased at minimum transmission point, and the MZM-c is applied to introduce phase difference of $\pi$ on the upper and lower branch, therefore $V_{D C 1}=V_{D C 2}=V_{D C 3}=4 \mathrm{~V}$. When the modulation index is small enough, the influence of the high-order sidebands can be ignored, and ${ }_{ \pm 1}$ order sidebands are preserved. The output expression of the upper branch is:

$$
E_{\text {out }-a}=j \frac{1}{4} E_{\text {in }}(t) J_{1}\left(V_{R F 1}\right)\left(e^{j \omega_{1} t}+e^{j 3 \omega_{1} t}+e^{j 5 \omega_{1} t}+e^{-j \omega_{1} t}+e^{-j 3 \omega_{1} t}+e^{-j 5 \omega_{1} t}\right)
$$

The output expression of the lower branch is:

$$
E_{\text {out }-b}=-\frac{1}{4} j E_{\text {in }}(t) J_{1}\left(V_{R F 1^{*}}\right)\left(e^{j \omega_{1} t}+e^{j 3 \omega_{1} t}+e^{j 5 \omega_{1} t}+e^{-j \omega_{1} t}+e^{-j 3 \omega_{1} t}+e^{-j 5 \omega_{1} t}\right)
$$

Combine the upper and lower branch of the DPMZM, the expression of the output signal can be defined as:

$$
E_{\text {out }-D P M Z M}(t)=j \frac{1}{4} E_{\text {in }}(t)\left[J_{1}\left(V_{R F 1}\right)-J_{1}\left(V_{R F 1^{*}}\right)\right] \cdot\left(e^{j \omega_{1} t}+e^{j 3 \omega_{1} t}+e^{j 5 \omega_{1} t}+e^{-j \omega_{1} t}+e^{-j 3 \omega_{1} t}+e^{-j 5 \omega_{1} t}\right)
$$

In the actual simulation, the optical carrier cannot be completely suppressed. In order to suppress the optical carrier, it is necessary to adjust the limiter to change the amplitude of the lower-branch optical signal, so that $J 0\left(V_{R F 1}\right) \approx J 0\left(V_{R F 1^{*}}\right)$, and realize the suppression of the optical carrier without affecting other harmonic components. The output spectrum of DPMZM is shown in Figure 4.

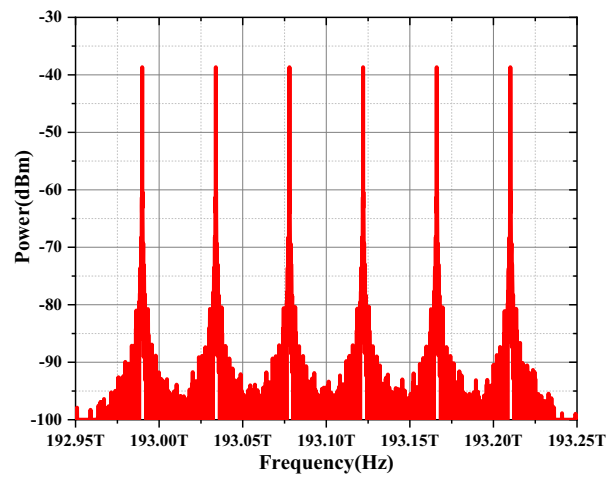

Figure 4. The optical spectrum at the output of DPMZM. 
On the upper branch of dual-parallel PolM, by adjusting PC1, the optical signal is injected into PolM at an angle of $45^{\circ}$ with the main axis of PolM. Assuming that the optical signal is $E_{i n}(t)$, which is injected into PolM, and the expression of RF is $V(t)=V \cos \omega t$. Set the angle between the Pol and the main axis of PolM as $\theta=45^{\circ}$, the output expression after Pol is:

$$
\begin{aligned}
E_{\text {out } \_ \text {pol }}(t) & =\frac{E_{\text {in }}(t)}{2}\left(\cos \theta \cdot \mathrm{e}^{j m \cos \omega t}+\sin \theta \cdot \mathrm{e}^{-j m \cos \omega t+j \varphi}\right) \\
& =\frac{E_{\text {in }}(t)}{2}\left\{\sum_{n=-\infty}^{\infty}\left[1-(-1)^{n} \mathrm{e}^{j \varphi}\right] j^{n} J_{n}(m) \mathrm{e}^{j n \omega t}\right\}
\end{aligned}
$$

Where $\varphi$ is the phase difference introduced by the upper and lower phase modulators, $m=\pi V / V_{\pi}$ is the modulation index of PolM, and $J_{n}(m)$ is the nth-order Bessel function. By adjust PC2, we can make the phase difference $\varphi=\pi$. If the value of ${ }_{m}$ is small, high-order Bessel function should be ignored, only the +1 order and -1 order harmonics are preserved. The output expression of the signal can be expressed as:

$$
E_{\text {out_pol }}(t)=j \frac{E_{\text {in }}(t)}{2} J_{1}(m)\left(\mathrm{e}^{j \omega t}+\mathrm{e}^{-j \omega t}\right)
$$

When RF signal is $V_{R F 2}(t)=V_{R F 2} \cos \left(\omega_{2} t\right)$, which is injected into the frequency multiplication circuit. The output signal of upper branch can be expressed as:

$$
E_{\text {upper }}(t)=j \frac{E_{\text {in }}(t)}{2} J_{1}\left(\frac{V_{R F 2}{ }^{5}}{16}\right)\left(\mathrm{e}^{j \omega_{2} t}+\mathrm{e}^{-j \omega_{2} t}+\mathrm{e}^{j 3 \omega_{2} t}+\mathrm{e}^{-j 3 \omega_{2} t}+\mathrm{e}^{j 5 \omega_{2} t}+\mathrm{e}^{-j 5 \omega_{2} t}\right)
$$

When RF signal is $V_{R F 2}^{*}(t)=V_{R F 2} \cos \left(2 \omega_{2} t\right)$, the output is:

$$
\begin{aligned}
E_{\text {lower }}(t) & =\frac{E_{\text {in }}(t)}{2}\left[J_{0}\left(V_{R F 2}\right)\left(1+\mathrm{e}^{j \varphi}\right)\right. \\
& +J_{1}\left(V_{R F 2}\right)\left(1+\mathrm{e}^{j \varphi}\right) \mathrm{e}^{j 2 \omega_{2} t}+J_{-1}\left(V_{R F 2}\right)\left(1+\mathrm{e}^{j \varphi}\right) \mathrm{e}^{-j 2 \omega_{2} t} \\
& \left.+J_{2}\left(V_{R F 2}\right)\left(1+\mathrm{e}^{j \varphi}\right) \mathrm{e}^{j 4 \omega_{2} t}+J_{-2}\left(V_{R F 2}\right)\left(1+\mathrm{e}^{j \varphi}\right) \mathrm{e}^{-j 4 \omega_{2} t}\right]
\end{aligned}
$$

If $J_{0}\left(V_{R F 2}\right)=J_{2}\left(V_{R F 2}\right)$ and $\cos \varphi=\left[J_{1}^{2}\left(V_{R F 2}\right)-J_{0}^{2}\left(V_{R F 2}\right)\right] /\left[J_{1}^{2}\left(V_{R F 2}\right)+J_{0}^{2}\left(V_{R F 2}\right)\right]$, by adjusting the $\varphi$, we can make $J_{0}\left(V_{R F 2}\right)=J_{ \pm 1}\left(V_{R F 2}\right)=J_{ \pm 2}\left(V_{R F 2}\right)$. When a beam of optical carrier is injected into PolM, the upper and lower output signals are shown in Figure 5.
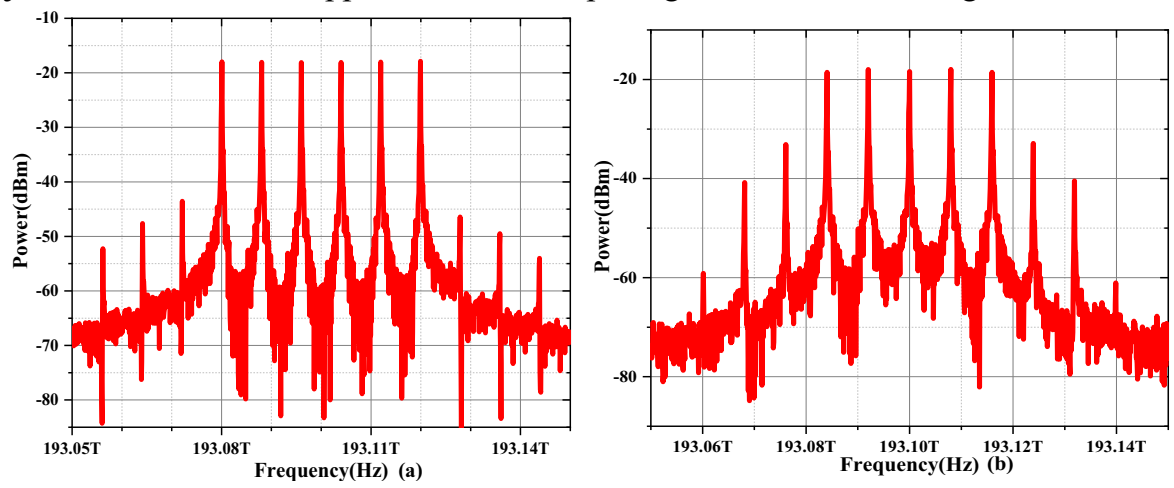

Figure 5. Optical spectrum at the output of upper (a) and lower(b) branch of dual-parallel PolM. 
On the upper branch of the dual-parallel PolM, each optical comb generated by DPMZM will be injected into PolM as an optical carrier for modulation. The output expression of the PolM1 is:

$$
\begin{aligned}
E_{P o l M 1}= & E_{\text {out-DPMZM }}(t) * \frac{1}{2} J_{1}\left(\frac{V_{R F 2}{ }^{5}}{16}\right) \cdot\left[\mathrm{e}^{j \omega_{2} t}+\mathrm{e}^{-j \omega_{2} t}+\mathrm{e}^{j 3 \omega_{2} t}+\mathrm{e}^{-j 3 \omega_{2} t}+\mathrm{e}^{j 5 \omega_{2} t}+\mathrm{e}^{-j 5 \omega_{2} t}\right] \\
= & j \frac{1}{8} E_{\text {in }}(t) J_{1}\left(\frac{V_{R F 2}{ }^{5}}{16}\right)\left[J_{1}\left(V_{R F 1}\right)-J_{1}\left(V_{R F 1^{1}}\right)\right] . \\
& \left(e^{j \omega_{1} t}+e^{j 3 \omega_{1} t}+e^{j 5 \omega_{1} t}+e^{-j \omega_{1} t}+e^{-j 3 \omega_{1} t}+e^{-j 5 \omega_{1} t}\right) . \\
& \left(e^{j \omega_{2} t}+\mathrm{e}^{-j \omega_{2} t}+\mathrm{e}^{j 3 \omega_{2} t}+\mathrm{e}^{-j 3 \omega_{2} t}+\mathrm{e}^{j 5 \omega_{2} t}+\mathrm{e}^{-j 5 \omega_{2} t}\right)
\end{aligned}
$$

On the lower branch of dual-parallel PolM, the OFC generated by DPMZM will be injected into an optical attenuator, by optical attenuator we can attenuate the input optical signal power. And then, each of optical comb line is injected into PolM as an optical carrier for modulation. The output expression of the PolM2 is:

$$
\begin{aligned}
E_{P O l M 2}= & E_{\text {out }-D P M Z M}(t) * \frac{1}{2} J_{0}\left(V_{R F 2}\right)\left(1+\mathrm{e}^{j \varphi}\right) \cdot\left(1+\mathrm{e}^{j 2 \omega_{2} t}+\mathrm{e}^{-j 2 \omega_{2} t}+\mathrm{e}^{j 4 \omega_{2} t}+\mathrm{e}^{-j 4 \omega_{2} t}\right) \\
= & j \frac{1}{8} E_{\text {in }}(t) J_{0}\left(V_{R F 2}\right)\left(1+\mathrm{e}^{j \varphi}\right)\left[J_{1}\left(V_{R F 1}\right)-J_{1}\left(V_{R F 1^{*}}\right)\right] . \\
& \left(e^{j \omega_{1} t}+e^{j 3 \omega_{1} t}+e^{j 5 \omega_{1} t}+e^{-j \omega_{1} t}+e^{-j 3 \omega_{1} t}+e^{-j 5 \omega_{1} t}\right) . \\
& \left(1+\mathrm{e}^{j 2 \omega_{2} t}+\mathrm{e}^{-j 2 \omega_{2} t}+\mathrm{e}^{j 4 \omega_{2} t}+\mathrm{e}^{-j 4 \omega_{2} t}\right)
\end{aligned}
$$

According to Eq.(9) and Eq.(10), we can get 36 comb lines and 30 comb lines respectively. By coupling the upper and lower branch, we can obtain 66-line OFC, and the expression can be expressed as:

$$
\begin{aligned}
E_{\text {out-DPPolM }}= & \left\{j \frac{1}{8} E_{\text {in }}(t) J_{1}\left(\frac{V_{R F}{ }^{5}}{16}\right)\left[J_{1}\left(V_{R F 1}\right)-J_{1}\left(V_{R F 1^{t}}\right)\right] .\right. \\
& \left(e^{j \omega_{1} t}+e^{j 3 \omega_{1} t}+e^{j 5 \omega_{1} t}+e^{-j \omega_{1} t}+e^{-j 3 \omega_{1} t}+e^{-j 5 \omega_{1} t}\right) . \\
& \left.\left(e^{j \omega_{2} t}+\mathrm{e}^{-j \omega_{2} t}+\mathrm{e}^{j 3 \omega_{2} t}+\mathrm{e}^{-j 3 \omega_{2} t}+\mathrm{e}^{j 5 \omega_{2} t}+\mathrm{e}^{-j \omega_{2} t}\right)\right\}+ \\
& \left\{j \frac{1}{8} E_{\text {in }}(t) J_{0}\left(V_{R F 2}\right)\left(1+\mathrm{e}^{j \omega}\right)\left[J_{1}\left(V_{R F 1}\right)-J_{1}\left(V_{R F 1^{*}}\right)\right] .\right. \\
& \left(e^{j \omega_{1} t}+e^{j 3 \omega_{1} t}+e^{j 5 \omega_{1} t}+e^{-j \omega_{1} t}+e^{-j 3 \omega_{1} t}+e^{-j 5 \omega_{1} t}\right) . \\
& \left.\left(1+\mathrm{e}^{j 2 \omega_{2} t}+\mathrm{e}^{-j 2 \omega_{2} t}+\mathrm{e}^{j \omega \omega_{2} t}+\mathrm{e}^{-j 4 \omega_{2} t}\right)\right\}
\end{aligned}
$$

In order to obtain an OFC with the same comb line interval, the frequencies of RF1 signal and RF2 signal need to satisfy the following relationship: $\omega_{1}=5.5 \omega_{2}$.

\section{Simulation results and analysis}

To prove that the scheme is feasible, we demonstrate the scheme by the simulation. First of all, a laser diode (LD) generates a continuous light carrier and the frequency of the light carrier is $193.1 \mathrm{THz}$, the power is $10 \mathrm{dBm}$, and the line-width is $10 \mathrm{MHz}$, which is modulated by DPMZM. The half-wave voltage of the MZM-a and MZM-b are set to $4 V$, the bias voltage of the upper and lower sub-MZMs are $V_{D C 1}=V_{D C 2}=4 \mathrm{~V}$, and the bias voltage of MZM-c is $V_{D C 3}=4 \mathrm{~V}$. The frequency of RF1 is $22 \mathrm{GHz}$, and the amplitude of $\mathrm{RF} 1$ is $1.0 \mathrm{~V}$. By adjusting the limiter, the amplitude of the input RF signal of the MZM$\mathrm{b}$ can be adjusted. In this scheme, adjusting the limiter to make the amplitude of the RF signal to $0.5 \mathrm{~V}$. The frequency of RF2 is $4 \mathrm{GHz}$, and the amplitude is $1.0 \mathrm{~V}$. On the upper branch of dual-parallel PolM, the frequency offset of the upper branch of PolM1 
is $90 \mathrm{deg}$, and the lower branch is $-90 \mathrm{deg}$. We set the rotation angle of PC1 to $45 \mathrm{deg}$, so that making the input signal and the main axis of PolM at the angle of $45 \mathrm{deg}$. Change the PC2 and setting its rotation angle to $45 \mathrm{deg}$, and the signal is injected into a Pol with a device angle of $45 \mathrm{deg}$. On the lower branch of dual-parallel PolM, the frequency of $\mathrm{RF}$ drive signal is $2 f_{R F 2}=8 \mathrm{GHz}$, the frequency offset of the upper branch of PolM2 is $210 \mathrm{deg}$, and the lower branch is $-210 \mathrm{deg}$. Similarly, the parameters of the PC3, PC4 and Pol are the same as the upper branch of dual-parallel PolM.

When the value of above parameters remain unchanged, as shown in Figure 6(a), the interval of the comb lines is $4 \mathrm{GHz}, 66$-line OFC is generated, the flatness is $0.73 \mathrm{~dB}$, and the SMSR is $14.19 \mathrm{~dB}$, the OSNR is about $29 \mathrm{~dB}$. When the frequency of the RF2 is $0.5 \mathrm{GHz}$, as shown in Figure 6(b), the interval of the comb lines is about $0.5 \mathrm{GHz}, 66$ line OFC is generated and the flatness is $1.16 \mathrm{~dB}$, and the SMSR is $13.69 \mathrm{~dB}$, the OSNR is about $19.5 \mathrm{~dB}$.
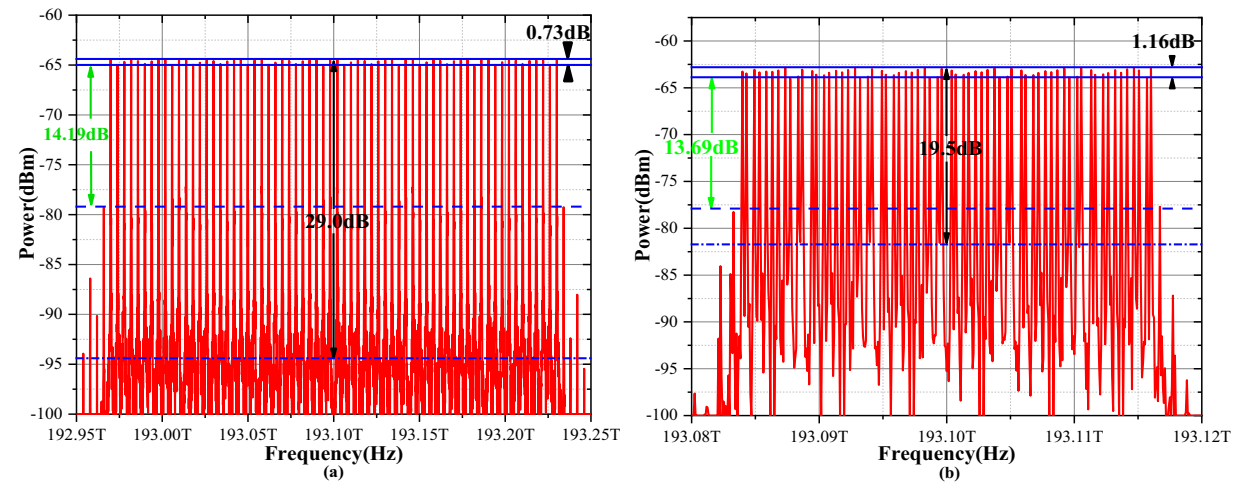

Figure 6. The generated optical comb with $66 \mathrm{comb}$ lines when the interval is $4 \mathrm{GHz}$ (a) and $0.5 \mathrm{GHz}$ (b).

According to Fig.6, we can generate 66 comb lines. When the frequency of RF2 is $4 \mathrm{GHz}$, the generated OFC is relatively flat, and the interval of the comb line is uniform. When the frequency of RF2 is $0.5 \mathrm{GHz}$, the generated OFC is also relatively flat, however, the interval of the comb line is not uniform, which is caused by the frequency multiplication circuit. The Fig.7 shows the comparison between the theoretical value and the simulated value of the output signal after different RF2 signals are injected into the frequency multiplication circuit.

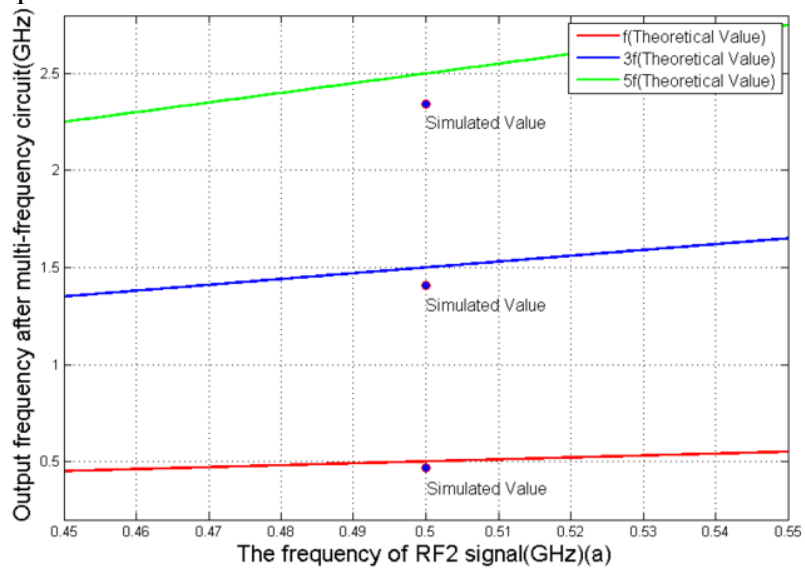




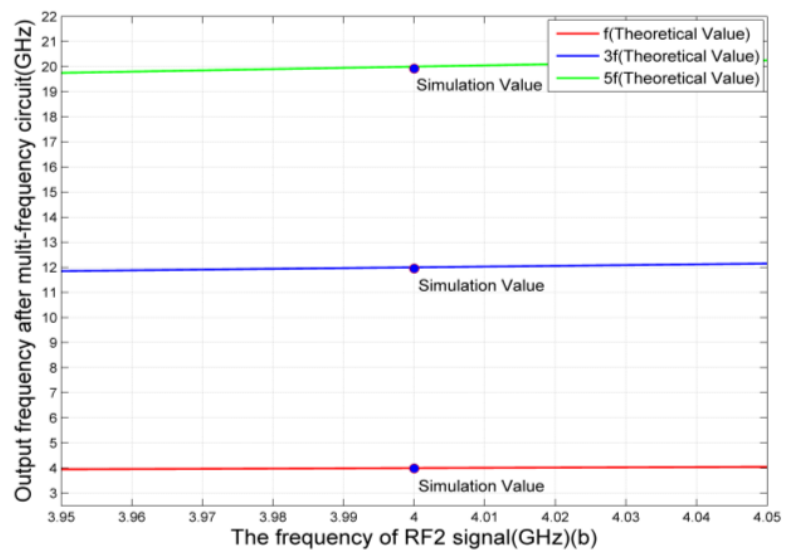

Figure 7. Comparison between the theoretical value and the simulated value of the output signal after $0.5 \mathrm{GHz}$ (a) and $4 \mathrm{GHz}$ (b) signals are injected into the frequency multiplication circuit.

As shown in Fig.7 (a), when the frequency of RF2 is $4 \mathrm{GHz}$, the simulated value of the output signal after frequency multiplication circuit is completely consistent with the theoretical value, and the output signal is $4 \mathrm{GHz}, 12 \mathrm{GHz}$ and $20 \mathrm{GHz}$. As shown in Fig.7 (b), when the frequency of RF2 is $0.5 \mathrm{GHz}$, the simulated value of the output signal after frequency multiplication circuit is not completely consistent with the theoretical value, and the output signal is $0.47 \mathrm{GHz}, 1.4 \mathrm{GHz}$ and $2.34 \mathrm{GHz}$, which causes the interval of the comb line is not so uniform.

\section{Conclusion}

In this scheme, an innovative scheme to generate the high-quality OFC based on DPMZM cascaded dual-parallel PolM with frequency multiplication circuit is proposed and demonstrated. In this scheme, when the interval of the comb lines is $4 \mathrm{GHz}, 66$ optical comb lines with a flatness of $0.73 \mathrm{~dB}$ is generated, and the SMSR is $14.19 \mathrm{~dB}$, the OSNR is about $30 \mathrm{~dB}$. When the frequency of the RF2 is $0.5 \mathrm{GHz}$, the interval of the comb lines is about $0.5 \mathrm{GHz}, 66$ optical comb lines with a flatness of $1.16 \mathrm{~dB}$ is generated, and the SMSR is $13.69 \mathrm{~dB}$, the optical signal noise ratio is about $21.14 \mathrm{~dB}$. In our proposal, by adjusting the frequency of the RF, we can adjust the interval of OFC. The results show the considerable approach for generation OFC. Compared with other schemes, this scheme requires low-power RF signal, and the minimum interval of comb lines can reach to about $0.5 \mathrm{GHz}$.

\section{Acknowledgment}

This work was supported by the National Nature Science Foundation of China (No.62161034, No.61561037, No.61861034, No.61763034).

\section{References}

[1] Yan, J., Xia, Z., Zhang, S., et al.: A flflexible waveforms generator based on a single dual-parallel MachZehnder Modulator. J. Opt. Commun. 334, 31-43 (2015). 
[2] W. Dai, W. Rao, H. Wang and H. Fu, "Microwave Photonic Filter by Using Recirculating Frequency Shifter to Generate Optical Frequency Comb," in IEEE Photonics Journal, vol. 13, no. 2, pp. 1-8, April 2021, Art no. 5500308, doi: 10.1109/JPHOT.2021.3069104.

[3] Atsushi Kanno, G. K. M. Hasanuzzaman, Naokatsu Yamamoto, Stavros Iezekiel, "Optical frequency comb applied optoelectronic oscillator for millimeter-wave signal generation and its application," Proc. SPIE 10634, Passive and Active Millimeter-Wave Imaging XXI, 106340E (8 May 2018); https://doi.org/10.1117/12.2304821.

[4] E. A. Anashkina and A. V. Andrianov, "Kerr-Raman Optical Frequency Combs in Silica Microsphere Pumped Near Zero Dispersion Wavelength," in IEEE Access, vol. 9, pp. 6729-6734, 2021, doi: 10.1109/ACCESS.2021.3049183.

[5] X. -Z. Li, M. Seghilani, L. R. Cortés and J. Azaña, "Sub-THz Optical Frequency Comb Generation by Efficient Repetition-Rate Multiplication of a 250-MHz Mode-locked Laser," in IEEE Journal of Selected Topics in Quantum Electronics, vol. 27, no. 2, pp. 1-10, March-April 2021, Art no. 7601310, doi: 10.1109/JSTQE.2020.3039280.

[6] Zhengyang Xie, Shangyuan Li, Haozhe Yan, Xuedi Xiao, Xiaoxiao Xue, Xiaoping Zheng, Bingkun Zhou, "Tunable ultraflat optical frequency comb generator based on optoelectronic oscillator using dual-parallel Mach-Zehnder modulator," Opt. Eng. 56(6) $066115 \quad$ (27 June 2017) https://doi.org/10.1117/1.OE.56.6.066115.

[7] K. Qu, S. Zhao, X. Li, Z. Zhu, D. Liang and D. Liang, "Ultra-Flat and Broadband Optical Frequency Comb Generator via a Single Mach-Zehnder Modulator," in IEEE Photonics Technology Letters, vol. 29, no. 2, pp. 255-258, 15 Jan.15, 2017, doi: 10.1109/LPT.2016.2640276.

[8] Cihai Chen, Chao He, Dan Zhu, Ronghui Guo, Fangzheng Zhang, and Shilong Pan, "Generation of a flat optical frequency comb based on a cascaded polarization modulator and phase modulator," Opt. Lett. 38, 3137-3139 (2013).

[9] Qiang Wang, Li Huo, Yanfei Xing, and Bingkun Zhou, "Ultra-flat optical frequency comb generator using a single-driven dual-parallel Mach-Zehnder modulator," Opt. Lett. 39, 3050-3053 (2014).

[10] Q. Chang, J. Gao and Y. Su, "Generation of optical comb frequency signal with high spectral flatness using two cascaded optical modulators," 2008 Asia Optical Fiber Communication \& Optoelectronic Exposition \& Conference, 2008, pp. 1-3. Yan, J., Xia, Z., Zhang, S., et al.: A flexible waveforms generator based on a single dual-parallel Mach-Zehnder Modulator. J. Opt. Commun. 334, 31-43 (2015). 